\title{
Análisis de corrosión en aceros con recubrimientos impacta en la competitividad en la industria metalmecánica de Mexicali
}

\author{
Corrosion analysis in coated steels impacts competitiveness \\ in the metalmecanic industry of Mexicali
}

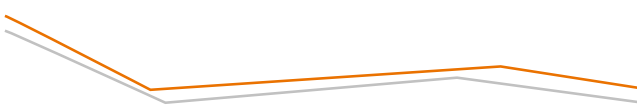

Gustavo López Badilla1*, César Sánchez Ocampo', Judith Marisela Paz Delgadillo', Juan Carlos Ling López

López Badilla, G., Sánchez Ocampo, C., Paz Delgadillo, J. M., Ling López, J. C. Análisis de corrosión en aceros con recubrimientos impacta en la competitividad en la industria metalmecánica de Mexicali. Investigación y Ciencia de la Universidad Autónoma de Aguascalientes. Número 69: 39-46, septiembre-diciembre 2016.
\end{abstract}

\section{RESUMEN}

Se realizó un análisis del uso de tres metales principales como lo son el cobre, cromo y níquel, que se aplican como proceso de deposición para protección de aceros ante la presencia de ambientes agresivos en interiores de la industria metalmecánica ubicada en Mexicali y que fabrica productos de acero. Aun con la aplicación de los métodos de cobreado, cromado y niquelado, en los procesos de manufactura se presentaba el proceso de corrosión en menor grado. El análisis se realizó en el año 2013 en la empresa señalada donde los factores principales de la generación de corrosión fueron la humedad y temperatura como variables climáticas, aunadas a los niveles de bióxido de azufre $\left(\mathrm{SO}_{2}\right)$ como parámetro ambiental. Además, se evaluó la funcionalidad de la protección en los productos con aceros para evitar la generación de corrosión con análisis de microscopía de barrido electrónico (MBE).

\section{ABSTRACT}

An analysis was made of the use of three main metals such as copper, chromium and nickel, which are applied as a deposition process for the protection

Palabras clave: industria metalmecánica, materiales metálicos, corrosión atmosférica, clima y contaminación, costos.

Keywords: metallurgical industry, metallic materials, atmospheric corrosion, climate and pollution, costs.

\section{Recibido: 30 de junio de 2015, aceptado: 13 de mayo de 2016}

Universidad Politécnica de Baja California.

Autor para correspondencia:glopezbadilla@yahoo.com of steel in the presence of aggressive environments in the metalworking industry located in Mexicali and that manufactures products of steel. Even with the application of the methods of copper, chromium and nickel, in the manufacturing processes the process of corrosion was presented to a lesser degree. The analysis was carried out in 2013 in the company indicated where the main factors of the corrosion generation were the humidity and temperature as climatic variables, coupled with the levels of sulfur dioxide $\left(\mathrm{SO}_{2}\right)$ as an environmental parameter. In addition, the functionality of the protection in products with steels was evaluated to avoid the corrosion generation with scanning electron microscopy (MBE) analysis.

\section{INTRODUCCION}

Los materiales que conforman los tipos de acero son muy utilizados en operaciones donde se aplican fuerzas de gran escala y se requieren esfuerzos importantes para contrarrestarlas, como en estructuras de edificaciones, puentes y astilleros, principalmente (López Badilla et al., 2012b). La industria metalmecánica es una de las que generan mayores ganancias a países como México, que comercializa metales duros y blandos principalmente compuestos por hierro, cromo, cobre y níquel, en conjunto con el carbono (López Badilla et al., 2012a).

Etapas de fabricación en la industria metalmecánica La fundición de metales se desarrolla a gran escala en la industria metalmecánica, con procesos donde se transforman minerales metálicos en materiales de tipo comercial para obtener productos que se utilizan en la vida cotidiana. Los metales procesados 
se utilizan para fabricar componentes de máquinas, equipos, instrumentos y herramientas que son necesarios para otras industrias, así como para otros sectores de la economía (Reardon, 2011). Varios tipos de metales y aleaciones se utilizan como materia prima, incluso el laminado para manufacturar barras, tiras, perfiles ligeros, hojas o tubos (CEC, 2006). Los metales son moldeados por los siguientes procesos:

- Fundición. El material metálico es fundido y se vierte en un molde.

- Forja. El material metálico se forma utilizando el martillo.

- Rodamiento. El material metálico se pasa a través de rodillos sucesivamente más estrechos para crear una hoja.

- Revestimiento láser. El polvo metálico se sopla a través de un haz láser móvil y el metal fundido resultante alcanza un sustrato para formar un baño de fusión.

- Extrusión. Un metal caliente y maleable es forzado bajo presión a través de una boquilla, que da forma antes de que se enfríe.

- Sinterización. Un metal en polvo se calienta en un ambiente no oxidante después de haber sido comprimido en una matriz.

- Mecanizado. En este proceso se utilizan tornos, fresadoras, taladros y cortadora en frío para dar forma.

Los procesos de trabajo en frío, donde la forma del producto se ve alterada por un rodamiento, pueden aumentar la resistencia del producto por un proceso llamado endurecimiento de trabajo. Esta propiedad del acero crea defectos microscópicos en el metal, que resisten más cambios de forma. Existen varias formas de fundición en la industria y el mundo académico. Una amplia variedad de técnicas se utilizan para terminar metales (Zhang y Zirakian, 2015); entre ellas molienda y pulido, chorreado abrasivo y muchas otras de acabado y revestimiento de superficie (galvanoplastia, galvanización, tratamiento térmico, anodización, revestimiento en polvo y así sucesivamente).

\section{Materiales utilizados en la industria metalmecánica}

Los metales de ingeniería comunes incluyen al aluminio, cromo, cobre, hierro, magnesio, níquel, titanio y zinc, y son los más utilizados como aleaciones y desarrollo de materiales metálicos para esta industria. Se ha destinado mucho esfuerzo en la comprensión del sistema de aleación hierrocarbono, que incluye aceros y hierros fundidos
(Davids et al., 2004). Los aceros lisos de carbono son los que contienen esencialmente sólo carbono como elemento de aleación, son muy utilizados por su bajo costo, las aplicaciones de alta resistencia, y en operaciones donde el peso y la corrosión no son un problema. La fundición, que incluye hierro dúctil, es también parte del sistema hierro-carbono. El acero inoxidable o acero galvanizado se utiliza cuando es importante la resistencia a la corrosión (Bringas, 2004).

\section{Tipos de acero}

Los aceros son una gran familia de metales, con aleaciones de hierro en el que se mezcla con carbono y otros elementos suaves con diferentes porcentajes de carbono a nivel alto (de $0.45 \%$ a $1.5 \%$ ), medio (de $0.25 \%$ a $0.45 \%$ ) y bajo (0.35\%), aunque nunca es mayor de $1.5 \%$ (Zirakian y Zhang, 2015). El acero es una aleación principalmente de hierro y carbono que se utiliza ampliamente en la construcción y otras aplicaciones debido a su fuerza, dureza y resistencia a la tracción. El carbono en aleaciones de acero típico podrá destinar hasta $2.1 \%$ de su peso atómico. La variación de la cantidad de elementos de aleación genera la forma del acero, ya sea como elementos de soluto o fases como precipitados, lo que retarda el movimiento de las dislocaciones que hacen que el hierro sea dúctil y débil y, por tanto, controla la dureza, ductilidad y resistencia a la fuerza de tracción. La fuerza de acero en comparación con el hierro puro solamente es posible por la ductilidad, por tener exceso de hierro (Fernández Miranda, 2006).

\section{Propiedades de los aceros}

Las propiedades de un acero están estrechamente relacionadas con su composición. Por ejemplo, existe una gran diferencia de dureza entre el acero en una lata de bebida y el utilizado para fabricar tijeras (ASTM, 2000). El rápido enfriamiento con agua fría de un acero cuando está al rojo vivo genera que sea más frágil, por lo que se debe enfriar lentamente. El tratamiento térmico es otro método que la siderúrgica utiliza para hacer que las propiedades del acero coincidan con lo requerido según el tipo de industria (CEC, 2006).

\section{Corrosión en aceros}

La corrosión es la ruina gradual de materiales (normalmente metales) por exposición a ambientes agresivos o no agresivos y la generación de una reacción química con su entorno (López Badilla et al., 2012b). Los metales pueden reaccionar con 
algún tipo de contaminante del aire o con el oxígeno que causa una oxidación electroquímica, es muy propensa en los aceros y produce óxidos de hierro principalmente.

La corrosión modifica las propiedades fisicoquímicas de los aceros y promueve su deterioro, lo que en ocasiones conlleva consecuencias fatales. Muchas aleaciones estructurales de acero se corroen simplemente por la exposición a la humedad en el aire, pero el proceso puede ser fuertemente afectado por la exposición a ciertas sustancias contaminantes.

\section{Procesos de recubrimiento en aceros como protección contra la corrosión}

Los metales son los materiales que más se utilizan para las plantas industriales, casas, edificios, puentes, automóviles y electrodomésticos. A medida que se exponen a ambientes agresivos de zonas áridas como el de la ciudad de Mexicali (López Badilla et al., 2012a) se genera corrosión en interiores de empresas. Esto debido a la presencia de sulfuros como principal agente poluto originario de la central geotérmica con el sulfuro de hidrógeno $\left(\mathrm{H}_{2} \mathrm{~S}\right)$ y derivados del azufre como el bióxido de azufre $\left(\mathrm{SO}_{2}\right)$ surgido del tráfico vehicular y lagunas de oxidación ubicadas en algunas áreas de la ciudad. Para garantizar la calidad y protección de productos de acero y la estabilidad de ganancias del mercado, las industrias metalmecánica y de otras áreas en Mexicali han utilizado diferentes tipos de recubrimientos metálicos (López Badilla et al., 2012b). Existen dos factores determinantes de los revestimientos de metal, uno es para la protección contra corrosión por la aplicación de una capa de construcción firme y el otro es la capa de acabado decorativo apropiado (Zirakian y Zhang, 2015). Los recubrimientos metálicos más comunes y populares son (ASTM, 2000):

a) Cobreado. El recubrimiento con cobre es el proceso en el que una película de dicho material se deposita sobre el elemento metálico que se desee proteger mediante el uso de una corriente eléctrica. Existen tres tipos básicos de procesos de cobreado disponibles comercialmente basados en procedimientos complejos

1. Baño alcalino. Se realiza con soluciones de cianuro.

2. Baño con ácido. Se lleva a cabo con sulfatos y fluoruroboratos.

3. Baño con fosfato puro. Se elabora con un ligero baño con sustancias alcalinas. b) Cromado. Este proceso se utiliza con la técnica de galvanoplastia, al depositar una película delgada de protección en el metal a proteger; la película de cromado puede ser para aspecto decorativo o de resistencia a la corrosión, para facilitar los procedimientos de limpieza o aumentar la dureza de la superficie. El proceso de cromado incluye las siguientes etapas:

1. Actividad de limpieza manual. Se desarrolla para eliminar todas las trazas residuales de la suciedad y las impurezas de la superficie dejadas por la operación con el desengrasante.

2. Diversos pretratamientos. Se lleva a cabo dependiendo del sustrato a aplicar para la deposición del cromo.

3. Colocación del recubrimiento. Se realiza la deposición del cobre en el material a proteger a alta temperatura cercana a los $150^{\circ} \mathrm{C}$.

4. Conductividad eléctrica. Se le suministra una corriente eléctrica en el orden de 10 microamperios.

c) Niquelado. En este proceso se utiliza la galvanoplastia para realizar los depósitos con películas muy finas sobre los metales a proteger. La película de níquel puede ser para decoración, resistencia a corrosión o al desgaste, antes que nada. La galvanoplastia de níquel es un proceso de deposición de este material sobre una pieza de metal, cuyos fragmentos para ser recubiertos deben esta limpios y libres de suciedad, corrosión y defectos de las placas. Para limpiar y proteger la pieza durante el proceso de recubrimiento se utilizan métodos de tratamiento térmico, limpieza y decapado, principalmente. Una vez que la pieza ha sido preparada se sumerge en una solución de electrolitos y se utiliza como cátodo. El ánodo de níquel se disuelve en el electrolito en forma de iones de níquel. Los iones viajan a través de la solución y se depositan sobre el cátodo.

\section{Posprocesamiento y costos de fabricación con materiales para recubrimientos metálicos}

El uso de los metales utilizados en esta investigación para procesos de recubrimiento de aceros fabricados como artículos manufacturados en la ciudad de Mexicali se basa en varios factores y uno de importancia es su precio (CEC, 2006). Este aspecto considerado en la manufactura implica una técnica adicional para dar mayor protección contra la corrosión a los productos de acero y se une a los costos de fabricación en cada metal protector. 
Acerca de los precios de los metales utilizados en esta investigación, el del cobre es de $3.2 \mathrm{dls} / \mathrm{lb}$, el cromo 1.2dls/lb y, por último, el níquel $8.6 \mathrm{dls} / \mathrm{lb}$. A estos precios se agregan los costos de operación de recubrimiento dependiendo del área a cubrir, el cromo realiza el proceso más rápido, después el cobre y, por último, el níquel.

\section{MATERIALES Y METODOS}

La exposición de aceros a procesos electroquímicos en periodos de humedad y temperatura mayores a $80 \%$ y $30^{\circ} \mathrm{C}$, aunados a niveles de concentración de sulfuros principalmente, en la ciudad de Mexicali, requiere recubrimientos que brinden la protección adecuada para evitar la corrosión. Esto ha conllevado utilizar varios tipos de depósitos metálicos para proteger los aceros y con ello mantenerlos mayor tiempo con vida. Además, se consideró que en algunos lugares cerrados de la empresa evaluada con procesos de manufactura se generaron ambientes agresivos por la presencia de sulfuros con niveles mayores a los permitidos por los estándares de calidad del aire. Dichos estándares son regulados por la Secretaría de Medio Ambiente y Recursos Naturales en México (SEMARNAT) y la Environmental Protection Agency (EPA) en Estados Unidos. De acuerdo con esto se desarrolló la investigación en 2013 en la empresa de metalmecánica instalada en Mexicali para determinar con base en ensayos experimentales el tipo de recubrimiento (cobre, cromo o níquel) al considerar el tipo y gastos del proceso para proteger los aceros, además de los costos de los metales usados como depósitos de protección. A continuación se mencionan las operaciones elaboradas:

- Se evaluaron las técnicas de la empresa referidas a niveles térmicos y se verificó si los índices de humedad y temperatura mayores a $80 \%$ y 30 ${ }^{\circ} \mathrm{C}$ tenían un efecto en al menos una pequeña variación en la temperatura de los hornos o fases de fabricación que alteraran algún tipo de característica de los aceros manufacturados. Los niveles de concentración del bióxido de azufre $\left(\mathrm{SO}_{2}\right)$ y óxidos de nitrógeno $\left(\mathrm{NO}_{\mathrm{x}}\right)$ se obtuvieron con el analizador de gases Lancom III-AMETEK.

- Se determinaron los costos de los metales usados como depósitos de protección con base en la afluencia de los mercados y la cantidad de compraventa para ser utilizados, además de los gastos generados en los procesos de fabricación de la empresa evaluada.

- Se realizaron microanálisis de los aceros con base en los estándares ASTM (2000) con los tres tipos de recubrimientos para determinar el de mayor protección y correlacionarlo con los costos del metal y de fabricación.

\section{RESULTADOS}

La presencia de corrosión en la industria metalmecánica de Mexicali genera gastos extra de los materiales al protegerlos del alto nivel de la misma, propia de la región. Esto incrementa los costos de producción en estas empresas y en compañías de ciudades con climas similares.

\section{Análisis de procesos de manufactura en empresa metalmecánica}

La mayoría de las etapas de fabricación de la empresa evaluada con el giro metalmecánico contienen procesos complejos, una es la de horno de hogar abierto (usado por el alto punto de fusión de acero), arco eléctrico (utilizado mediante convenios de empresas que suministran energía eléctrica) y de refinación (usado para oxidar con oxígeno a alta presión el carbono y otros elementos para obtener el acero). Las etapas en específico son las siguientes:

Con base en las etapas del diagrama de la Figura 1 se realizaron análisis de correlación de productos fabricados en buen estado y defectuosos, costos de fabricación considerando al personal, además de equipos y máquinas de la empresa evaluada. Estos factores mencionados se relacionaron con los

\begin{tabular}{|c|c|c|c|c|}
\hline $\begin{array}{l}\text { Introducción a } \\
\text { hornos de } \\
\text { minerales: hierro, } \\
\text { carbono de coque } \\
\text { y piedra caliza }\end{array}$ & $\begin{array}{l}\text { Disminución } \\
\text { del alto } \\
\text { contenido de } \\
\text { carbono }\end{array}$ & $\begin{array}{l}\text { Tipo de } \\
\text { colada }\end{array}$ & $\begin{array}{l}\text { Obtención } \\
\text { de } \\
\text { productos } \\
\text { de acero }\end{array}$ & $\begin{array}{l}\text { Uso de } \\
\text { recubrimientos: } \\
\text { Cu, } \\
\text { Cry Ni }\end{array}$ \\
\hline
\end{tabular}

Figura 1. Ełapas de fabricación del acero. Elaboración propia. 
índices de humedad relativa (HR) y temperatura y con los niveles de concentración de $\mathrm{SO}_{2}$ y $\mathrm{NO}_{x}$, como se muestra en la Tabla 1. Ahí se observa que en los periodos de invierno disminuyen los niveles de producción e incrementan los productos defectuosos; mientras que los costos requeridos para trabajos de tiempo extra por artículos defectivos se incrementan en mayor proporción en la misma época. El rendimiento operativo del personal, además de los equipos y maquinaria industrial en época invernal fue mayor que en la veraniega y los índices de humedad relativa se presentaron con mayor intensidad en dicho periodo, así como los niveles de concentración de los contaminantes evaluados, y los índices de temperatura fueron mayores en la época de verano.

Aunado al análisis de producción con cobre, cromo y níquel, usados en las etapas de manufactura de los recubrimientos como materia prima, se colocaron los gastos de procesos de fabricación con los métodos de protección de los aceros, como se menciona en la Tabla 2. En esta evaluación se consideraron las cantidades en gramos del producto de deposición de cada metal en el acero, además de los periodos requeridos en el proceso del recubrimiento de cada metal.
La Tabla 2 muestra el efecto que tiene el proceso de recubrimiento en cada metal con una figura geométrica redonda, de acuerdo a sus características físicas y químicas en correlación con los precios del procedimiento de deposición. Conforme se incrementa el diámetro del área a cubrir y el espesor del recubrimiento aumentan la cantidad en gramos del metal a cubrir, tiempo de deposición y costos por el proceso de recubrimiento en cada metal. También se desarrolló un análisis para materiales con figura geométrica cuadrada, se pueden observar los valores en la Tabla 3.

\section{Microanálisis de aceros con recubrimientos}

En los análisis a nivel microscópico se muestra el deterioro de los materiales de cobre, cromo y níquel utilizados como muestras experimentales; se observa en las zonas de color blanco la presencia de derivados del azufre y en los lugares un poco oscuros los óxidos de nitrógeno.

La Figura 2 muestra el daño que se genera en los recubrimientos que protegen los aceros fabricados en la industria metalmecánica de Mexicali, y que son afectados por variaciones climáticas aun siendo mínimas (en ocasiones menores a $5 \%$ y $1{ }^{\circ} \mathrm{C}$ ). Esto ocasiona una preocupación al personal que utiliza

Tabla 1. Correlación de producción, costos, clima y polución del aire en empresa de Mexicali (2013)

\begin{tabular}{|c|c|c|c|c|c|c|c|c|c|c|}
\hline \multirow{2}{*}{$\begin{array}{c}\text { FACTORES } \\
\text { MESES }\end{array}$} & \multirow{2}{*}{$\begin{array}{l}\text { CPF } \\
\text { TAM }\end{array}$} & \multirow{2}{*}{$\begin{array}{l}\text { CPD } \\
\text { TAM }\end{array}$} & \multicolumn{2}{|c|}{$\begin{array}{c}\text { ICTE } \\
\%\end{array}$} & \multirow{2}{*}{$\begin{array}{c}\text { ROP } \\
\%\end{array}$} & \multirow{2}{*}{$\begin{array}{c}\text { ROEYMI, } \\
\%\end{array}$} & \multicolumn{2}{|c|}{ CLIMA } & \multicolumn{2}{|c|}{ POLUCIÓN } \\
\hline & & & $\begin{array}{c}\text { MO, } \\
\%\end{array}$ & $\begin{array}{c}\text { EyM, } \\
\%\end{array}$ & & & $\begin{array}{l}\text { HR } \\
\%\end{array}$ & $\begin{array}{l}{ }^{\mathrm{T}} \\
{ }^{\circ} \mathrm{C}\end{array}$ & $\begin{array}{l}\mathrm{SO}_{2} \\
\mathrm{ppm}\end{array}$ & $\begin{array}{l}\mathrm{NO}_{\mathrm{x}} \\
\mathrm{ppb}\end{array}$ \\
\hline Enero & 1153 & 82 & 16 & 14 & 83 & 74 & 87 & 13 & 0.78 & 79 \\
\hline Febrero & 1192 & 65 & 13 & 12 & 78 & 72 & 82 & 18 & 0.66 & 71 \\
\hline Marzo & 1284 & 51 & 11 & 11 & 74 & 65 & 76 & 23 & 0.54 & 65 \\
\hline Abril & 1341 & 37 & 8 & 9 & 72 & 63 & 70 & 26 & 0.49 & 54 \\
\hline Mayo & 1374 & 43 & 6 & 7 & 70 & 60 & 67 & 32 & 0.43 & 47 \\
\hline Junio & 1296 & 55 & 5 & 6 & 67 & 57 & 72 & 34 & 0.38 & 43 \\
\hline Julio & 1284. & 59 & 6 & 7 & 62 & 54 & 84 & 42 & 0.41 & 49 \\
\hline Agosto & 1273 & 64 & 7 & 8 & 58 & 50 & 89 & 40 & 0.47 & 56 \\
\hline Septiembre & 1378 & 70 & 9 & 9 & 66 & 56 & 82 & 35 & 0.51 & 62 \\
\hline Octubre & 1356 & 74 & 10 & 11 & 73 & 62 & 77 & 29 & 0.59 & 69 \\
\hline Noviembre & 1297 & 79 & 11 & 13 & 76 & 66 & 81 & 22 & 0.63 & 74 \\
\hline Diciembre & 1279 & 86 & 14 & 15 & 80 & 70 & 88 & 14 & 0.72 & 84 \\
\hline
\end{tabular}

CPF (TAM). Cantidad fabricada (t de acero por mes), CPD (TAM). Cantidad de productos defectuosos (t de acero por mes), ICTE (\%). Incremento de costos por tiempo extra (\%) - MO (Mano de obra) - EyMI. (Equipo y maquinaria industrial), ROP (\%). Rendimiento operativo del personal (\%) - Cantidad de piezas fabricadas contra errores cometidos, ROEyMI (\%). Rendimiento operativo de los equipos y maquinaria industrial (\%) Cantidad de piezas fabricadas contra fallas eléctricas y mecánicas, HR (\%). Humedad relativa (\%), T ( $\left.{ }^{\circ} \mathrm{C}\right)$. Temperatura $\left({ }^{\circ} \mathrm{Celsius}\right), \mathrm{SO}_{2}(\mathrm{ppm})$. Bióxido de azufre, NOx (ppb). Óxido de nitrógeno. Elaboración propia. 
Tabla 2. Correlación de costos, proceso y tiempo de deposición de cada metal redondo (2013)

\begin{tabular}{|c|c|c|c|c|c|c|c|c|c|c|}
\hline \multirow{2}{*}{\multicolumn{2}{|c|}{$\begin{array}{c}\text { Metales } \\
\text { Especificaciones } \\
\text { del recubrimiento }\end{array}$}} & \multicolumn{3}{|c|}{ Cobre } & \multicolumn{3}{|c|}{ Cromo } & \multicolumn{3}{|c|}{ Níquel } \\
\hline & & \multirow[t]{2}{*}{ M.gr } & \multirow[t]{2}{*}{$t$} & \multirow[t]{2}{*}{ C } & \multirow[t]{2}{*}{ M.gr } & \multirow[t]{2}{*}{$t$} & \multirow[t]{2}{*}{ C } & \multirow[t]{2}{*}{ M.gr } & \multirow[t]{2}{*}{$t$} & \multirow[t]{2}{*}{ C } \\
\hline $\mathrm{D}, \mathrm{cm}$ & $\mathrm{e}, \mathrm{mm}$ & & & & & & & & & \\
\hline 2.00 & 0.0025 & 0.14 & 1426 & 0.95 & 0.11 & 4191 & 1.17 & 0.14 & 1532 & 1.43 \\
\hline 2.50 & 0.0030 & 0.21 & 2139 & 1.22 & 0.17 & 6288 & 1.30 & 0.21 & 2298 & 1.67 \\
\hline 3.00 & 0.0035 & 0.30 & 2994 & 1.45 & 0.24 & 8803 & 1.44 & 0.29 & 3218 & 1.88 \\
\hline 3.50 & 0.0040 & 0.39 & 3993 & 1.63 & 0.32 & 11737 & 1.59 & 0.39 & 4290 & 2.11 \\
\hline 4.00 & 0.0045 & 0.50 & 5133 & 1.79 & 0.41 & 15091 & 1.72 & 0.50 & 5515 & 2.34 \\
\hline 4.50 & 0.0050 & 0.63 & 6417 & 1.88 & 0.51 & 18863 & 1.88 & 0.63 & 6895 & 2.78 \\
\hline 5.00 & 0.0055 & 0.77 & 7843 & 2.07 & 0.62 & 23055 & 1.99 & 0.77 & 8427 & 3.13 \\
\hline 5.50 & 0.0060 & 0.93 & 9411 & 2.13 & 0.75 & 27666 & 2.23 & 0.92 & 10112 & 3.67 \\
\hline 6.00 & 0.0065 & 1.10 & 11122 & 2.22 & 0.88 & 32696 & 2.85 & 1.09 & 11951 & 4.23 \\
\hline 6.50 & 0.0070 & 1.30 & 12976 & 2.33 & 1.03 & 38146 & 3.67 & 1.27 & 13943 & 4.89 \\
\hline
\end{tabular}

D. Diámetro en cm de una pieza de acero a cubrir para su protección; e. Espesor en mm del recubrimiento; M.gr. Gramos del metal a cubrir; t. Tiempo en segundos en que se realiza la deposición, C. Costo en pesos por cada m recubierto del proceso de deposición. Elaboración propia.

Tabla 3. Correlación de costos, proceso y tiempo de deposición de cada metal cuadrado (2013)

\begin{tabular}{|c|c|c|c|c|c|c|c|c|c|c|}
\hline \multirow{2}{*}{\multicolumn{2}{|c|}{$\begin{array}{c}\text { Metales } \\
\text { Especificaciones } \\
\text { del recubrimiento }\end{array}$}} & \multicolumn{3}{|c|}{ Cobre } & \multicolumn{3}{|c|}{ Cromo } & \multicolumn{3}{|c|}{ Níquel } \\
\hline & & \multirow[t]{2}{*}{ M.gr } & \multirow[t]{2}{*}{$t$} & \multirow[t]{2}{*}{ C } & \multirow[t]{2}{*}{ M.gr } & \multirow[t]{2}{*}{$t$} & \multirow[t]{2}{*}{ C } & \multirow[t]{2}{*}{ M.gr } & \multirow[t]{2}{*}{$t$} & \multirow[t]{2}{*}{ C } \\
\hline $\mathrm{A}, \mathrm{cm}^{2}$ & $\mathrm{e}, \mathrm{mm}$ & & & & & & & & & \\
\hline 4.00 & 0.0025 & 0.089 & 908 & 1.23 & 0.072 & 2669 & 1.33 & 0.089 & 975 & 1.78 \\
\hline 6.25 & 0.0030 & 0.17 & 1702 & 1.34 & 0.135 & 5004 & 1.46 & 0.167 & 1929 & 1.96 \\
\hline 9.00 & 0.0035 & 0.28 & 2859 & 1.53 & 0.226 & 8406 & 1.65 & 0.280 & 3073 & 2.23 \\
\hline 12.25 & 0.0040 & 0.44 & 4448 & 1.76 & 0.352 & 13076 & 1.78 & 0.436 & 4780 & 2.56 \\
\hline 16.00 & 0.0045 & 0.65 & 6535 & 1.92 & 0.517 & 19214 & 1.92 & 0.641 & 7023 & 2.88 \\
\hline 20.25 & 0.0050 & 0.91 & 9191 & 2.13 & 0.728 & 27020 & 2.23 & 0.901 & 9876 & 3.43 \\
\hline 25.00 & 0.0055 & 1.23 & 12482 & 2.22 & 0.989 & 36693 & 2.45 & 1.223 & 13412 & 3.78 \\
\hline 30.25 & 0.0060 & 1.63 & 16476 & 2.33 & 1.305 & 48435 & 2.78 & 1.615 & 17704 & 4.17 \\
\hline 36.00 & 0.0065 & 2.09 & 21242 & 2.42 & 1.682 & 62445 & 2.95 & 2.082 & 22825 & 4.56 \\
\hline 42.25 & 0.0070 & 2.65 & 26847 & 2.55 & 2.126 & 78924 & 4.24 & 2.632 & 28848 & 5.67 \\
\hline
\end{tabular}

A. Área en $\mathrm{cm}^{2}$ de una pieza de acero con lado por lado, a cubrir para su protección; e. Espesor en mm del recubrimiento; M.gr. Gramos del metal a cubrir; t. Tiempo en s en que se realiza la deposición, C. Costo en pesos por cada m recubierto del proceso de deposición. Elaboración propia.

este tipo de productos de acero en la región y en otras zonas del país, donde se usan para edificaciones; la mayor concentración está en la ciudad de Mexicali para la industria de la construcción que requiere materiales reforzados por ser zona sísmica. La Figura 2 es un microanálisis de la época de verano. En la Figura 2a se observa el mayor daño en el recubrimiento de cobre que puede proteger al acero por ciertos periodos de vida de los mismos, pero posteriormente el ataque del fenómeno corrosivo es en los aceros y es cuando se ocasionan graves consecuencias. En la
Figura $2 b$ que representa el recubrimiento de cromo se muestra un daño menor al del cobre, pero con la posibilidad de que en un futuro corto se genere lo mismo que en la Figura 2a. La Figura 2c, que indica el recubrimiento de níquel, tiene un menor grado de deterioro.

La Figura 3 representa el microanálisis de la época invernal. Es similar a la Figura $2 a$ para el recubrimiento de cobre, pero con un grado de deterioro mayor; una posible causa de esto es la 


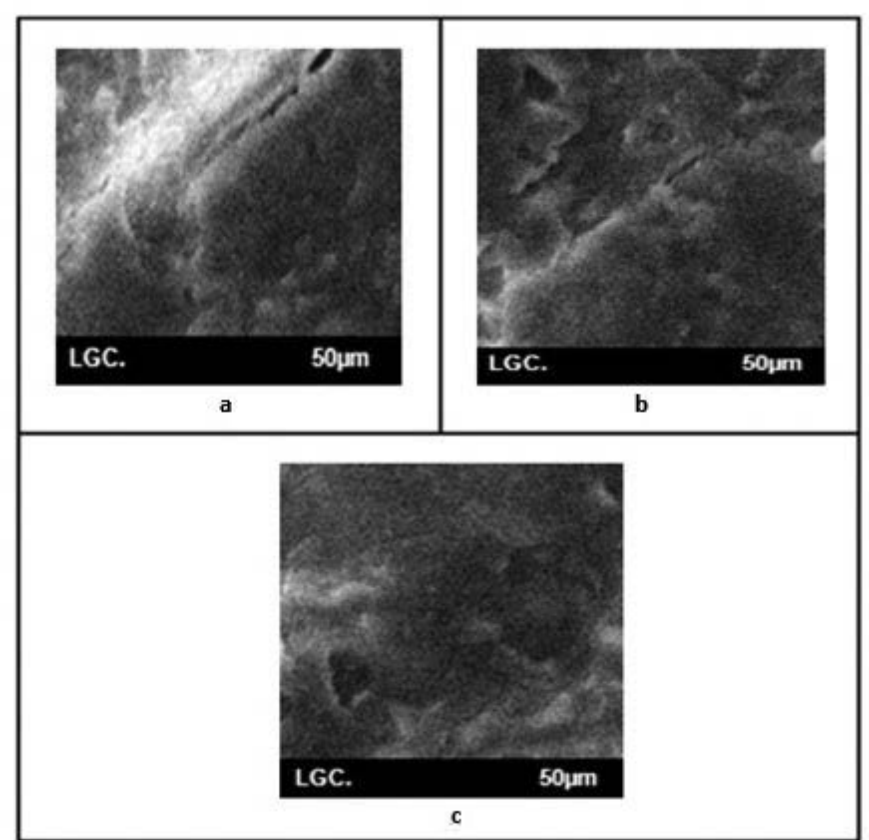

Figura 2. Microanálisis del deterioro de los recubrimientos depositados en aceros de (a) cobre, (b) cromo y (c) níquel en la época de verano en la industria metalmecánica de Mexicali. Fotografías tomadas por los autores.

presencia de altos niveles de humedad. Lo mismo ocurre con la Figura $3 b$, a diferencia de la $2 b$ con el recubrimiento de cromo, donde se observa como mancha blanca una mayor concentración de derivados del azufre en un área de las probetas experimentales y el grado de deterioro es mayor. La Figura $3 c$ indica para el recubrimiento de níquel daño principalmente en el área de color blanca de la muestra experimental, pero también tiende a expandirse el daño a varias zonas del metal.

\section{DISCUSION}

La presencia de corrosión atmosférica en la industria metalmecánica de la ciudad de Mexicali genera grandes pérdidas económicas a este tipo de empresas, donde las variaciones de los niveles de humedad y temperatura, aunadas a las concentraciones de $\mathrm{SO}_{2}$ y $\mathrm{NO}_{x}$ que sobrepasan los niveles estándar, son la principal causa del fenómeno corrosivo. Esto afecta la productividad y durabilidad de los productos manufacturados de este tipo de compañías en esta región de la República Mexicana y el uso de recubrimientos puede proteger contra la corrosión; aunque esto implica un proceso adicional en la fabricación que redunda en tiempos y costos de fabricación, lo que afecta con ello la competitividad a nivel nacional e internacional, causa en ocasiones pérdida de empleos, baja en la producción y a veces en microempresas o de nivel medio, el cierre de las mismas. Con base en lo anterior se elaboró el estudio, donde se obtuvo la información para fabricar un nuevo material con los metales especificados anteriormente, como el de menor costo y mejor funcionalidad.

\section{CONCLUSIONES}

El estudio mostró que con el uso de cada elemento por separado (cobre, cromo y níquel) se genera en la superficie del acero al carbono una película protectora, pero en este tipo de acero se origina el fenómeno de corrosión en ambientes corrosivos, como los que se presentan en algunos periodos del año en la empresa evaluada. La aplicación del revestimiento de cobre, cromo y níquel aleados en conjunto, determinó que es necesario utilizar este material propuesto para reducir al mínimo o evitar la corrosión en el acero al carbono que sufría graves deterioros antes del proceso experimental. Uno de los aspectos de importancia fue el análisis de costos con la evaluación de la contaminación del aire y

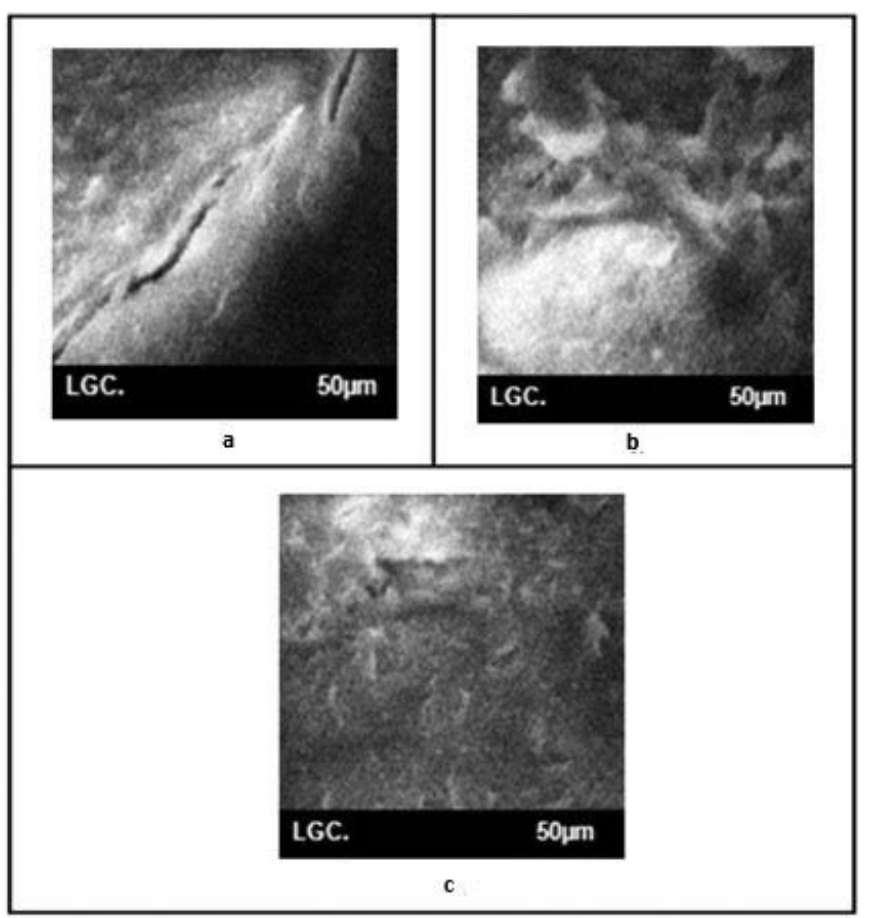

Figura 3. Microanálisis del deterioro de los recubrimientos depositados en aceros de (a) cobre, (b) cromo y (c) níquel en la época de invierno en la industria metalmecánica de Mexicali. Fotografías tomadas por los autores. 
clima, e indica que los metales propuestos tienen bajo costo, son afines al hierro y carbono y que con los mismos se reduce el proceso de corrosión en los aceros al carbono.

\section{Agradecimientos}

El personal de investigación de la Universidad Politécnica de Baja California agradece a la empresa de la industria metalmecánica que permitió el desarrollo de la investigación.

\section{LITERATURA CITADA}

- ASTM Standards. Standards to technical experiments. 2000.

- BRINGAS, J. E. (Ed.). Handbook of Comparative World Steel Standards. 3 ed. (p. 14). PA, US: ASTM International, 2004.

- COMISSION OF THE EUROPEAN COMMUNITIES (CEC) (2006). Analysis of economic indicators of the EU metals industry: the impact of raw materials and energy supply on competitiveness. Commission Staff Working Document. Brussels, 2.8.2006, SEC (2006) 1069, 2006.

- DAVIDS, K. et al. Practical guide to use stainless steels. Materials Performance, 4(3): 58-66, 2004.

- FERNÁNDEZ MIRANDA, Y. Preparación mecánica de minerales y carbones. Editorial Dossat, S. A., 2006.

- LÓPEZ BADILLA, G. et al. Atmospheric corrosion in the seafood industry in the northwwest of México. Cientifica, 16(2): 67-73, $2012 a$.
- LÓPEZ BADILLA, G. et al. Micro and nano corrosion in steel cans used in the seafood industry. En B. Valdez (Ed.), Scientific, Health and Social Aspects of the Food Industry. INTECH Publisher, 2012b.

- ZHANG, J. Y ZIRAKIAN, T. Probabilistic assessment of structures with SPSW systems and LYP steel infill plates using fragility function method. Engineering Structures, 85, 195-205, 2015.

- $\quad$ ZIRAKIAN, T. y ZHANG, J. Structural performance of unstiffened Iow yield point steel plate shear walls. Journal of Constructional Steel Research, 112, 40-53, 2015.

\section{De páginas electrónicas}

- REARDON, A. C. (Ed.). Metallurgy for the non-metallurgist. 2 ed. Ohio, US: The Materials Information Society-ASM International, 2011. ISBN 978-1-61503-821-3. Recuperado de http://www. asminternational.org/documents/10192/3476320/05306G_ TOC.pdf/07678056-70c3-4c2f-910d-33fc7e8dd2c5 